\title{
CORRIGENDA
}

\section{The comprehensive ICF core sets for spinal cord injury from the perspective of physical therapists: a worldwide validation study using the Delphi technique}

KH Herrmann, I Kirchberger, G Stucki and A Cieza

Spinal Cord (2011) 49, 676; doi:10.1038/sc.2011.26

Correction to: Spinal Cord advance online publication, 23 November 2010; doi:10.1038/sc.2010.155

Since the publication of the above article, the authors have identified an error.
The title for Table 6 should read 'Health conditions and personal factors: percent agreement among participants in the final Delphi round', and not 'ICF personal factors: ...'.

The authors would like to apologise for this error.

\section{The comprehensive ICF core sets for spinal cord injury from the perspective of occupational therapists: a worldwide validation study using the Delphi technique}

KH Herrmann, I Kirchberger, G Stucki and A Cieza

Spinal Cord (2011) 49, 676; doi:10.1038/sc.2011.27

Correction to: Spinal Cord advance online publication, 30 November 2010; doi:10.1038/sc.2010.168

Since the publication of the above article, the authors have identified an error.
The title for Table 6 should read 'Responses that were linked to health conditions and personal factors: percent agreement among participants in the final Delphi round', and not 'Responses that were linked to the ICF component personal factors:...'.

The authors would like to apologise for this error. 